\title{
(2) OPEN ACCESS Four-year observation of the changes in corneal
endothelium cell density and correlated factors after
Implantable Collamer Lens V4c implantation
}

\author{
Wen Yang, ${ }^{1}$ Jing Zhao, ${ }^{2}$ Ling Sun, ${ }^{2}$ Jiao Zhao, ${ }^{3}$ Lingling Niu, ${ }^{2}$ Xiaoying Wang, ${ }^{2}$ \\ Xingtao Zhou (i) ${ }^{2}$
}

${ }^{1}$ Department of Ophthalmology, The Third People's Hospital of Chengdu, The Affiliated Hospital of Southwest Jiaotong University, Chengdu, China 'Department of Ophthalmology, Eye and ENT Hospital of Fudan University; NHC Key Laboratory of Myopia; Laboratory of Myopia, Chinese Academy of Medical Sciences, Shanghai, China

${ }^{3}$ Department of Ophthalmology, People's Hospital of Leshan, Leshan, China

Correspondence to Dr Xingtao Zhou, Department of Ophthalmology, Eye and ENT Hospital of Fudan University; NHC Key Laboratory of Myopia; Laboratory of Myopia, Chinese Academy of Medical Sciences, Shanghai, China;

doctzhouxingtao@163.com

WY and IZ contributed equally.

WY and JZ are joint first authors.

Received 24 February 2020 Revised 11 May 2020

Accepted 25 May 2020

Published Online First

16 June 2020

\section{ABSTRACT}

Background To evaluate the changes in corneal endothelium cell density (ECD) and the correlated factors after Implantable Collamer Lens (ICL) V4c implantation. Methods In this retrospective, consecutive study, 48 eyes of 25 patients with myopia who underwent ICL V4c implantation were enrolled. Patients were followed up for at least 4 years, during which manifest refraction, uncorrected distance visual acuity, corrected distance visual acuity (CDVA), intraocular pressure, ECD, anterior chamber depth, anterior chamber volume (ACV), anterior chamber angle (ACA), vault and distance from the corneal endothelium to the central ICL (C-ICL) were measured. Spearman's correlation analysis was used to identify variables correlated with changes in ECD, and generalised estimating equation model adjusting withinpatient intereye correlations was used to predict changes in ECD.

Results All surgeries were performed safely with no complications during follow-up (average $52 \pm 2.9$ months). Safety and efficacy indices were $1.23 \pm 0.22$ and $1.04 \pm 0.16$, respectively. No eyes had decreased CDVA, and $67 \%$ gained one or more lines. Further, $79 \%$ were within $\pm 0.50 \mathrm{D}$, and $100 \%$ were within $\pm 1.0 \mathrm{D}$ of the attempted refraction. Additionally, a $4.03 \% \pm 2.2 \%$ reduction in ECD compared with the preoperative value was observed at the last follow-up visit. Changes in ECD were significantly correlated with vault, $\mathrm{C}-\mathrm{ICL}$, change in ACA and change in ACV. Vault was the most significant factor for changes in ECD.

Conclusions ICL V4c implantation is safe and effective for myopia correction. Anterior segment biometric parameters including the vault, ACA and C-ICL may influence changes in ECD; specifically, the vault plays a major role.

\section{INTRODUCTION}

The EVO Implantable Collamer Lens (ICL; Model V4c; STAAR Surgical, Monrovia, California, USA) is a single-piece posterior chamber phakic intraocular lens designed with a central port. Since it became commercially available in 2011 , it has been shown to be a safe and effective way to correct myopia. ${ }^{1}$ ICL $\mathrm{V} 4 \mathrm{c}$ has eliminated the prior procedure of iridotomy or iridectomy which is required by ICL implantation. $^{2}$ The central hole helps the aqueous flowing from the posterior chamber to the anterior chamber, which maintains the normal physiology of the anterior segment of the eye. Kawamorita et $a l^{3}$ demonstrated that the $0.36 \mathrm{~mm}$ central port increases aqueous flow by using computational fluid dynamics technology and discovered a decreased risk of anterior subcapsular cataracts.

Corneal endothelium cells are important in maintaining the transparency of the cornea. The loss of corneal endothelium cells after intraocular surgery, especially lens surgery, may cause dysfunction and lead to corneal oedema. ${ }^{4}$ The new central port design of the ICL V4c changes the aqueous flow, and may therefore influence corneal endothelium cells. Reports $^{5-7}$ have described that endothelium cell density (ECD) loss ranges from 2\% to $9 \%$ after ICL and ICL V4c implantation. However, few reports have focused on the correlations between various factors and ECD loss after ICL V4c implantation. Therefore, evaluation of the corneal ECD changes and analysis of the correlated factors require further investigation. In this study, we aimed to observe the long-term changes in ECD after ICL V4c implantation and to investigate the correlated factors.

\section{MATERIALS AND METHODS \\ Subjects}

This was a retrospective, consecutive and observational study. Patients between the ages of 20 and 40 (mean age, $28.85 \pm 5.83$ ) years who underwent ICL V4c implantation at the Department of Eye \& ENT Hospital of Fudan University in Shanghai, China, from February 2015 to October 2015 were included. Inclusion criteria were as follows: age of 20-40 years, stable refractive error and no contact lens use $\geq 2$ weeks. Exclusion criteria were as follows: ocular disease such as corneal degeneration or dystrophy, lens opacity, retinal disease and uncontrolled glaucoma, a history of ocular inflammation or trauma, previous ocular surgeries, a history of systemic diseases and ECD $<2000$ cells/ $\mathrm{mm}^{2}$. All patients underwent routine preoperative examinations.

\section{Examinations}

Preoperative examinations included: (1) uncorrected distance visual acuity (UDVA), corrected distance visual acuity (CDVA), spherical equivalent (SE), slit lamp examination and funduscopic examinations; (2) intraocular pressure (IOP) was measured with a non-contact tonometer (Canon Full Auto Tonometer TX-F; Canon, Tokyo, Japan); (3) anterior chamber depth (ACD), corneal thickness, corneal diameter (white to white), anterior chamber volume (ACV) and anterior chamber angle 
(ACA) were measured with a Pentacam HR (Oculus Optikgerate Wetzlar, Germany); and (4) ECD was measured by non-contact specular microscopy (SP-2000P, Topcon Corporation, Japan).

\section{Operation procedure}

Three days before surgery, antibiotic eye drops were prescribed four times daily. All surgeries were performed by two experienced surgeons (XZ, XW) with the same technique. After topical anaesthesia was administered, a hole ICL was inserted through a $3.0 \mathrm{~mm}$ clear corneal incision after sodium hyaluronate was placed in the anterior chamber. The hyaluronate was then washed out using a balanced salt solution after the ICL was placed in the posterior chamber. The details of the procedure have been described by Chen et al. ${ }^{8}$ After the surgery, patients were prescribed antibiotic and steroid eye drops four times per day within 2 weeks.

\section{Follow-up}

All patients were followed up for at least 4 years. The postoperative examinations including UDVA, CDVA, refractive power, IOP, ECD and anterior segment measurements were performed by a skilled examiner. Another examiner who was blinded to the patients' information overviewed the diagram and measured the vault and the distance between central ICL and corneal endothelium (C-ICL) values using the image analysis software program accompanying the Pentacam device. The ECD was measured three times, as the value varied within \pm 30 cells; it was considered to have good repeatability. The postoperative values at 3 months and at the last follow-up were included in the analysis, as these two time points represent early and long-term outcomes, respectively.

\section{Statistical analysis}

All statistical analyses were performed using the IBM Statistical Package for the Social Sciences V.23.0. Data are expressed as the mean $\pm S D$. The Shapiro-Wilk test was used to determine if the variables accord with normal distribution. The paired t-test was used for normally distributed data, while the Wilcoxon signedrank test was used for non-normally distributed ones. Repeated measurement analysis using generalised estimating equation (GEE) model to adjust for intereye dependency was applied to compare the differences in measurement data before and after the operation. Spearman correlation analysis was used to identify relationships between the changes in ECD and vault, C-ICL, the change in ACA, ACV and ACD. GEE model adjusting withinpatient intereye correlations was employed to predict the change in ECD. $\mathrm{P}$ values $<0.05$ were considered statistically significant.

\section{RESULTS}

Forty-eight eyes of 25 patients underwent ICL V4c implantations. All surgeries and postoperative follow-ups were uneventful, and no complications such as infection, bleeding or ocular hypertension were noted. The mean follow-up duration was $52.06 \pm 2.88$ months (range, 48-57 months). The clinical parameters and biometric values of the patients before and after ICL implantation ( 3 months postoperatively and the last follow-up) are shown in table 1.

\section{Safety and efficacy}

At the last follow-up, the safety index (postoperative CDVA/ preoperative CDVA) was $1.23 \pm 0.22$ (range, 1-1.75), and the efficacy index (postoperative UDVA/preoperative CDVA) was $1.04 \pm 0.16$ (range, 0.8-1.5). Among all of the eyes, $83 \%$ had
Table 1 The clinical parameters and biometric values of the patients before and after the Implantable Collamer Lens implantation

\begin{tabular}{llll}
\hline Parameters & Preoperative & 3-month follow-up & The last follow-up \\
\hline UDVA (Logmar) & $1.30 \pm 0.36$ & $-0.04 \pm 0.09^{*} \dagger$ & $0.02 \pm 0.09^{*} \dagger$ \\
Range (min, max) & $(0.8-2.0)$ & $(-0.2$ to 0.2$)$ & $(-0.2$ to 0.2$)$ \\
CDVA (Logmar) & $0.01 \pm 0.1$ & $-0.09 \pm 0.07^{*}$ & $-0.09 \pm 0.09^{*}$ \\
Range (min, max) & $(-0.1$ to 0.4$)$ & $(-0.2$ to 0.1$)$ & $(-0.2$ to 0.09$)$ \\
SE (D) & $-10.11 \pm 3.03$ & $0.10 \pm 0.35^{*} \dagger$ & $-0.20 \pm 0.32^{*} \dagger$ \\
Range (min, max) & $(-20.5 \ddagger$ to -5.5$)$ & $(-0.47$ to 0.88) & $(-0.69$ to 0.52$)$ \\
ACD (mm) & $3.14 \pm 0.22$ & $2.97 \pm 0.26^{*}$ & $2.96 \pm 0.22^{*}$ \\
Range (min, max) & $(2.80-3.67)$ & $(2.42-3.57)$ & $(2.61-3.51)$ \\
IOP (mm Hg) & $14.45 \pm 2.58$ & $13.25 \pm 2.51$ & $13.73 \pm 1.86$ \\
Range (min, max) & $(8.3-20.1)$ & $(9.2-18.3)$ & $(10.9-18.7)$ \\
\hline ACV ( $\mu$ L) & $194.71 \pm 27.29$ & $115.52 \pm 24.30^{*}$ & $116.6 \pm 20.15^{*}$ \\
Range (min, max) & $(154-261)$ & $(75-163)$ & $(84-165)$ \\
ACA ( $\left.{ }^{\circ}\right)$ & $37.30 \pm 4.08$ & $23.34 \pm 5.12^{*} \dagger$ & $24.24 \pm 4.41^{*} \dagger$ \\
Range (min, max) & $(28.7-44.8)$ & $(11.10-33.8)$ & $(15.7-33.3)$ \\
ECD (cells/mm²) & $2913 \pm 169$ & $2963 \pm 95 \dagger$ & $2795 \pm 166^{*} \dagger$ \\
Range (min, max) & $(2403-3135)$ & $(2705-3106)$ & $(2322-3007)$ \\
\hline
\end{tabular}

*Versus preoperative, $\mathrm{p}<0.05$.

†3-month follow-up versus the last follow-up, $p<0.05$.

$\ddagger$ The preoperative refractive power of this eye was $-18.75 \mathrm{DS} /-3.50 \mathrm{DC} \times 15^{\circ}$. The spherical power was completely corrected and the attempted SE was $-2.50 \mathrm{D}$ (-0.75 DS/-3.50 DC $\left.\times 15^{\circ}\right)$.

$A C A$, anterior chamber angle; $A C D$, anterior chamber depth; $A C V$, anterior chamber volume; CDVA, corrected distance visual acuity; ECD, endothelium cell density; IOP, intraocular pressure; SE, epherical equivalent; UDVA, uncorrected distance visual acuity.

a postoperative UDVA of $20 / 20$ or better. Further, $100 \%$ had a postoperative UDVA of 20/40 or better. One hundred per cent postop UDVA was the same or better than preoperative CDVA. No eyes had decreased CDVA, and 67\% of the eyes gained one or more lines; $27 \%$ gained two or more lines (figure $1 \mathrm{~A}-\mathrm{C}$ ).

\section{Predictability and stability}

The attempted versus achieved SE corrections is shown in a scatter plot (figure 1D). At the last follow-up, 79\% of the eyes were within $\pm 0.50 \mathrm{D}$; none of the eyes were beyond $\pm 1.0 \mathrm{D}$ of the attempted refraction (figure $1 \mathrm{E}$ ). The average SE powers were $0.10 \pm 0.35 \mathrm{D}$ and $-0.20 \pm 0.32 \mathrm{D}$ at the 3 -month follow-up and at the last follow-up session, respectively. There were statically significant differences in UDVA and SE between the two postoperative follow-ups. The refraction remained stable during the follow-up period (figure $1 \mathrm{~F}$ ).

\section{Anterior biometric parameters}

There was a significant difference in the values of ACD, ACV and ACA between the preoperation and postoperation measurements (both at 3 months and at the last follow-up). There was no significant difference in the value of IOP between the preoperation and the follow-up measurements (repeated measurement analysis, $\mathrm{p}>0.05)$. The ACA at the 3 -month follow-up was significantly lower than the last follow-up $(p<0.05)$.

\section{Vault of ICL}

Three months after the operation and at the last follow-up, the mean values of vault were $424.72 \pm 205.74$ and $401.25 \pm 196.48 \mu \mathrm{m}$, respectively, which were not significantly different (Wilcoxon signed-rank test, $\mathrm{p}=0.059$ ). Of all the eyes, $67 \%$ (33 eyes) were between $250 \mu \mathrm{m}$ and $750 \mu \mathrm{m}$. Further, $22.9 \%(11 / 48)$ of the eyes were between $150 \mu \mathrm{m}$ and $250 \mu \mathrm{m}$ (no eyes were below $150 \mu \mathrm{m})$, and $4 \%(2 / 48)$ of the eyes were 


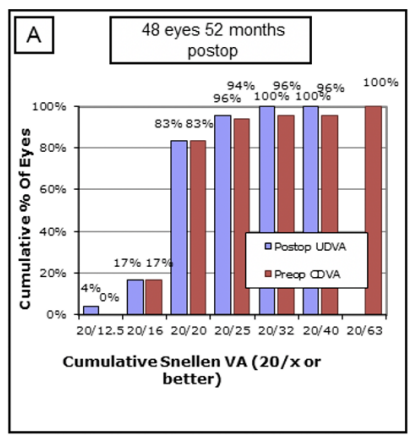

Uncorrected Distance Visual Acuity

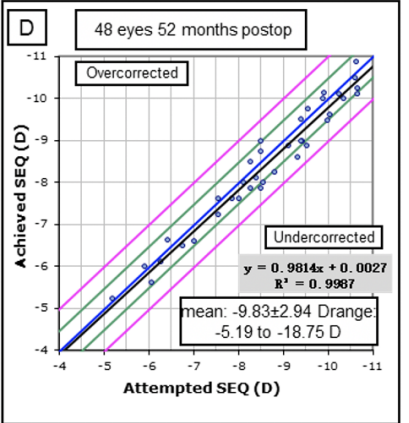

Spherical Equivalent Refraction Attempted vs Achieved

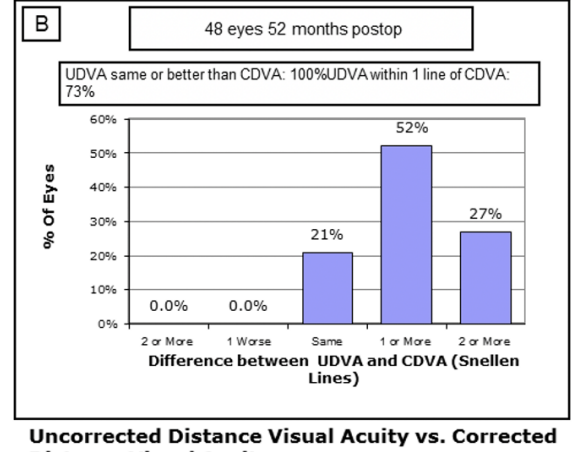

Distance Visual Acuity

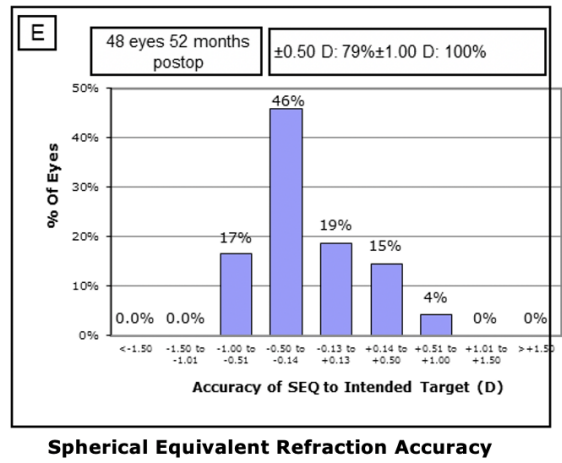

Spherical Equivalent Refraction Accuracy

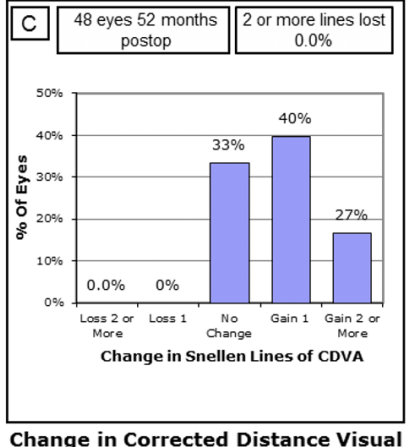

Change in Corrected Distance Visual Acuity

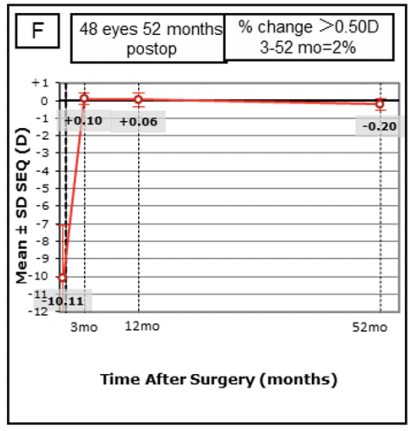

Spherical Equivalent Refraction Stability

Figure 1 Clinical outcomes of 48 eyes with myopia at the last follow-up after the implantation of Implantable Collamer Lens V4c. (A) Postoperative uncorrected distance visual acuity (UDVA) versus preoperative corrected distance visual acuity (CDVA). (B) Difference between postoperative UDVA and preoperative CDVA. (C) Change in CDVA. (D) Attempted spherical equivalent refraction change versus the achieved spherical equivalent refraction change. (E) Distribution of postoperative spherical equivalent refraction accuracy. (F) Stability of spherical equivalent refraction up to 52 months. D, diopters; mo, months; Postop, postoperative; Preop, preoperative; SEQ, spherical equivalent.

between $900 \mu \mathrm{m}$ and $920 \mu \mathrm{m}$. Fortunately, no cataracts or high IOPs were observed among the eyes below or above the ideal vault range.

\section{The distance between central ICl and corneal endothelium}

Three months after the operation and at the last follow-up visit, the mean values of C-ICL were $2428.33 \pm 248.10 \mu \mathrm{m}$ and $2425.21 \pm 246.17 \mu \mathrm{m}$, respectively; this difference was nonsignificant (paired t-test, $\mathrm{p}=0.143$ ).

\section{Corneal endothelium density}

Preoperatively, as well as 3 months after the operation and at the last follow-up, the mean values of ECD were $2913 \pm 169$ cells $/ \mathrm{mm}^{2}, \quad 2963 \pm 95$ cells $/ \mathrm{mm}^{2}$ and $2795 \pm 166 \mathrm{cells} / \mathrm{mm}^{2}$, respectively. No significant difference was observed between the preoperative ECD value and the value at 3 months. At the last follow-up, the ECD was reduced by $4.03 \% \pm 2.20 \%$ compared with the preoperative value $(\mathrm{p}<0.001)$. However, no eyes decreased to less than $2000 \mathrm{cell} / \mathrm{mm}^{2}$ or had a significant loss over $30 \%$.

There were significant correlations between the $\triangle \mathrm{ECD}$ (preoperative ECD-last follow-up ECD) and the vault $(\mathrm{r}=0.715$, $\mathrm{p}<0.001)$, C-ICL $(\mathrm{r}=-0.285, \mathrm{p}<0.05), \Delta$ ACA (preoperative ACA-last follow-up ACA; $r=0.590, \mathrm{p}<0.001$ ) and $\triangle \mathrm{ACV}$ (preoperative ACV-last follow-up ACV; $\mathrm{r}=0.539, \mathrm{p}<0.01$; figure 2). $\mathrm{ACD}$ and $\triangle \mathrm{ACD}$ (preoperative ACD-last follow-up ACD) were not correlated with $\triangle E C D(p=0.509$ and $p=0.456)$. Six explanatory variables were used in the GEE model for prediction of the $\triangle \mathrm{ECD}$. The vault, C-ICL and $\triangle \mathrm{ACA}$ was significantly associated with $\triangle \mathrm{ECD}$ and the vault was most accountable for the ECD loss (table 2).

\section{DISCUSSION}

Corneal endothelium cells have remained an important concern for patients undergoing intraocular procedures, especially anterior segment surgeries, such as lens surgery. We retrospectively evaluated patients who underwent ICL V4c implantation at our hospital when it was first introduced in China. Overall, this study aimed to investigate the long-term safety of the surgery and the long-term changes in ECD as well as the correlated factors after ICL V4c implantation.

As this study demonstrated, the efficacy index was $1.04 \pm 0.16$ and the safety index was $1.23 \pm 0.22$ at the last follow-up visit, which was similar to previous studies. ${ }^{9-12}$ Specifically, Miao et $\mathrm{al}^{9}$ reported that the efficacy index was $1.14 \pm 0.23$, and the safety index was $1.33 \pm 0.22$ at 3 months after ICL V4c implantation for moderate-to-high myopia. Further, Yan et $a l^{10}$ reported that the efficacy index was $1.03 \pm 0.23$, and the safety index was $1.24 \pm 0.26$ after 2 years postoperatively. In this study, there was favourable safety, efficacy and stability of ICL V4c implantation; $67 \%$ of the eyes gained at least one line. In addition, $77 \%$ of the eyes remained within $\pm 0.50 \mathrm{D}$ after 4 years. Although there was a statically significant difference in UDVA and SE between the two postoperative follow-ups, there was no clinical significance for this small variance. Alfonso et al ${ }^{11}$ reported that $19.1 \%$ of the eyes gained one or more lines, and that $95 \%$ of the eyes were within $\pm 0.50 \mathrm{D}$ after 6 months, while the mean preoperative SE was less than $-9 \mathrm{D}$. In the present study, the mean preoperative SE was more than $-10 \mathrm{D}$. Our results demonstrated the long-term efficacy and safety of ICL V4C implantation for patients with myopia.

At 3 months after surgery, the ECD value was $2963 \pm 95$ cells/ $\mathrm{mm}^{2}$. There was no significant difference compared with the 

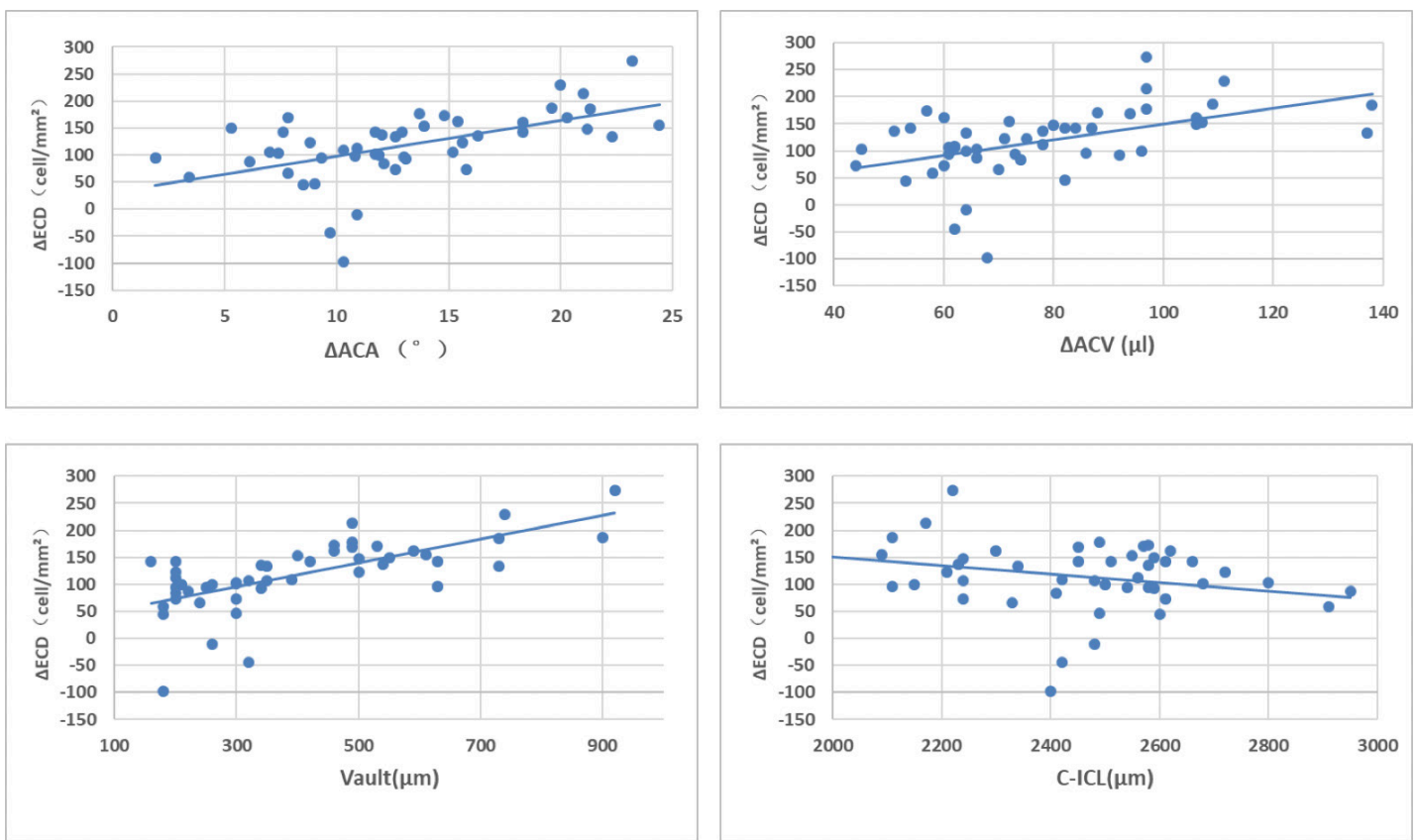

Figure 2 Correlations between the $\triangle E C D$ (preoperative endothelium cell density (ECD) - last follow-up $E C D$ ) and $\triangle A C A$ (preoperative anterior chamber angle (ACA)- last follow-up ACA), $\triangle \mathrm{ACV}$ (preoperative anterior chamber volume (ACV) - last follow-up ACV), vault and C-ICL. C-ICL, the distance between central ICL and corneal endothelium; $\triangle$, change in.

preoperative value. However, the ECD value was $2795 \pm 166$ cells/ $\mathrm{mm}^{2}$ at 4 years postoperatively, which reflected a $4.03 \% \pm 2.20 \%$ reduction compared with the preoperative ECD. Bhandari $e^{2} a l^{13}$ reported that the ECD reduction was $6.1 \%$ at 9 months after ICL V4c implantation, and Lee $e t a l^{14}$ reported a 7.8\% $\pm 8.3 \%$ reduction in ECD at least 5 years after ICL implantation. In another long-term study, Igarashi et $a l^{15}$ followed up patients after ICL implantation for 8 years and found that the ECD reduction was $6.2 \% \pm 8.6 \%$. Some studies have also specifically focused on ECD after ICL V4c implantation. In a prospective study including 147 eyes (80 subjects), Lisa et al ${ }^{16}$ reported a $1.7 \%$ reduction in ECD at 1 year after ICL V4c implantation. In a large multicentre case series, Kamiya et $\mathrm{al}^{17}$ found a mean decrease of $0.1 \% \pm 10 \%$ ECD reduction in 351 eyes from 7 institutes after 1 year follow-up. The $10 \% \mathrm{SD}$ in the former study was mainly because it was a multicentre study; further, the equipment and measuring methods were different between institutions. In comparison, the SD value was only $2.2 \%$ in our study, and all of the measurements were performed using the same instrument. Our results showed a similar or lower level of reduction of ECD loss compared with previous reports, and our follow-up period was longer (beyond 4 years), further verifying the long-term safety of ICL V4c implantation.

ECD loss may be composed of the acute loss caused by surgery and chronic loss such as physiological loss during the follow-up period. No significant reductions in ECD were observed at the 3 -month follow-up visit, which indicates that there was no acute ECD loss induced by the surgery. In other words, the ECD loss in this study was associated with chronic loss, including physiological loss, which was reported to decline by about $0.6 \%$ per year $^{18}$; thus, the accumulative total would be $2.4 \%$ at the last follow-up visit. We suspect that the decline in ECD may be induced by other factors which require further investigation.

In the present study, there was no significant difference in IOP at the follow-up visits compared with the preoperative value, which was similar to what was reported by Shimizu et al $^{19}$; they observed no IOP increase at the 1-year follow-up. In addition, no correlation between IOP and the $\triangle \mathrm{ECD}$ was detected.

The postoperative ACD values were smaller than the preoperative value, but there was no significant difference between the 3 -month value and that at the last follow-up. No correlation was detected between the $\triangle \mathrm{ECD}, \triangle \mathrm{ACD}$ or $\mathrm{ACD}$ at the last follow-up

Table 2 Factors predicting $\triangle E C D$ determined by generalised estimating equation model adjusting for within-patient intereye correlations

\begin{tabular}{|c|c|c|c|c|c|c|}
\hline \multirow[b]{2}{*}{ Independant variable } & \multirow[b]{2}{*}{ Mean \pm SD } & \multirow[b]{2}{*}{ Unstandardised coefficients } & \multirow[b]{2}{*}{ Standardised coefficients } & \multicolumn{2}{|l|}{$95 \% \mathrm{Cl}$} & \multirow[b]{2}{*}{$P$ value } \\
\hline & & & & Lower limit & Upper limit & \\
\hline Vault $(\mu \mathrm{m})$ & $401 \pm 196$ & 0.205 & 0.625 & 0.097 & 0.313 & 0.000 \\
\hline C-ICL $(\mu \mathrm{m})$ & $2420 \pm 246$ & 0.103 & 0.403 & 0.030 & 0.175 & 0.006 \\
\hline$\triangle \mathrm{ACA}\left({ }^{\circ}\right)$ & $78.10 \pm 21.59$ & 3.817 & 0.318 & 0.798 & 6.836 & 0.013 \\
\hline$\triangle \mathrm{ACV}(\mu \mathrm{L})$ & $0.18 \pm 0.15$ & 0.353 & 0.118 & -0.359 & 1.065 & 0.332 \\
\hline$\triangle \mathrm{ACD}(\mathrm{mm})$ & $13.06 \pm 5.36$ & 35.749 & 0.083 & -45.953 & 117.451 & 0.391 \\
\hline $\mathrm{ACD}(\mathrm{mm})$ & $29.96 \pm 0.22$ & 3.876 & 0.013 & -45.012 & 52.765 & 0.877 \\
\hline
\end{tabular}

Variables in the table body are ordered according to the strength of the contribution, which was based on the standardised coefficient.

$\Delta$ Variable=preoperative variable - postoperative variable (the last follow-up).

$A C A$, anterior chamber angle; $A C D$, anterior chamber depth; $A C V$, anterior chamber volume; $C-I C L$, the distance between corneal endothelium and central ICL; ECD, endothelium cell density. 
in the current study. Nevertheless, Saxena et $a l^{20}$ demonstrated that a shallower ACD was a predictor factor for ECD loss after Artisan lens implantation. In another study conducted at our institution, Niu et $^{2 l^{21}}$ reported that there was an $8.38 \% \pm 0.06 \%$ reduction in ECD among patients whose preoperative ACD was less than $2.8 \mathrm{~mm}$. The difference was mainly because the mean preoperative and postoperative 4 years $\mathrm{ACD}$ of the patients in this study was $3.14 \pm 0.22 \mathrm{~mm}$ and $2.96 \pm 0.22 \mathrm{~mm}$, respectively; no eyes with ACD less than $2.8 \mathrm{~mm}$ were included. Further investigation is needed to analyse the correlation between $\triangle \mathrm{ECD}$ and the postoperative shallow ACD less than $2.8 \mathrm{~mm}$.

In this study, there was a negative correlation between the C-ICL and the $\triangle E C D$. Several reports ${ }^{22-26}$ regarding the anterior chamber lens showed a decreased distance between the corneal endothelium and central ICL, which increased the loss of ECD. Two reports in 2017 emphasised the importance of C-ICL in reducing the loss of ECD. ${ }^{23}{ }^{24}$ Although there was no exact explanation of the mechanisms, we assume that the decreased C-ICL may increase the shear stress of the aqueous flow to the endothelium; however, the mechanisms need to be investigated further.

The ACV and ACA values after surgery were significantly decreased from the preoperative value and remained stable at the last follow-up. Although there was a significant decrease in ACA at the 3-month follow-up compared with the last follow-up, the change was mild and had no clinical significance. Both the $\triangle \mathrm{ACA}$ and the $\triangle \mathrm{ACV}$ were positively correlated with the $\triangle \mathrm{ECD}$ in the present study. Fernández-Vigo ${ }^{27}$ reported a similar result and demonstrated that the decrease in ACA may be caused by the haptic of the ICL V4c. We postulate that the changes in ACV and ACA may have altered the aqueous flow, which resulted in an insufficient metabolism of the corneal endothelial cells. However, further investigation is needed to verify this hypothesis.

The average vault at the last follow-up was $401.25 \pm 196.48 \mu \mathrm{m}$. Our result was consistent with some previous reports on ICL $\mathrm{V} 4 \mathrm{c}$. For example, Lisa $e \mathrm{al}^{16}$ reported a mean average vault of $405.5 \pm 184.7 \mu \mathrm{m}$ in 147 eyes after 1 year, and Lee et $\mathrm{l}^{28}$ found a mean average vault of $399.5 \pm 151.9 \mu \mathrm{m}$ in 54 eyes after 1 month. We found that there was a positive correlation between the vault and the $\triangle \mathrm{ECD}$. Multiple regression analysis indicated that the vault could play a major role in ECD changes. Excessively high vault values increased the risk of ECD loss and the occurrence of glaucoma. Conversely, excessively low vault values increased the risk of anterior subcapsular cataract. ${ }^{29} \mathrm{~A}$ few studies have focused on the range of vault for surgical safety. Gonvers $e t a l^{30}$ reported that a vault equal to or less than $90 \mu \mathrm{m}$ was a risk factor for the development of anterior subcapsular cataracts, and that $150 \mu \mathrm{m}$ should be regarded as the lower limit of safe vault. Zeng et $a l^{31}$ suggested a vault safety range from $100 \mu \mathrm{m}$ to $1000 \mu \mathrm{m}$. In our study, $67 \%$ of eyes were within the optimal range (between $250 \mu \mathrm{m}$ and $750 \mu \mathrm{m})$. Further, 11 eyes were between $150 \mu \mathrm{m}$ and $250 \mu \mathrm{m}$, and no eyes were below $150 \mu \mathrm{m}$. No instances of cataracts or glaucoma were observed during the follow-up period. According to the GEE model adjusting for within-patient intereye correlations, the present study found that the ECD loss was correlated with vault, C-ICL and $\triangle \mathrm{ACA}$, while the vault was the most significant factor to predict ECD loss. The result implies that we should monitor the ECD change attentively in eyes with high vault after ICL V4C implantation for the long-term safety.

This study has several limitations. First, the sample size was small. Second, the data for other time points were missing during the long follow-up period, as some patients did not attend the scheduled follow-up visits. Third, the age of the patients ranged from 20 to 40 years old; we did not divide them into different age groups. In the future, a study with a larger sample size with different age groups is desired to confirm the various correlation factors for ECD change after ICL V4c implantation.

In conclusion, ICL V4c implantation is a safe and effective procedure for myopia correction. The anterior segment biometric parameters including the vault, ACA and C-ICL may influence the change in ECD; however, the vault plays a major role.

Correction notice This paper has been updated since it was published online. The affiliations and correspondence address have been corrected.

Acknowledgements The authors would like to thank Editage (www.editage.cn) for English language editing.

\section{Collaborators None.}

Contributors The study concept and design; drafting of the manuscript and critical revision of the manuscript were done by WY, JZ and XZ. Data collection and analysis and interpretation of data were undertaken by WY, JZ, LS, LN and XW. Supervision was done by XZ. WY and IZ contributed equally to this work and should be considered as equal first authors.

Funding Supported in part by the National Natural Science Foundation of China (Grant no. 81770955); Joint research project of new frontier technology in municipal hospitals (SHDC12018103); the Project of Shanghai Science and Technology (Grant no.17411950200); the National Natural Science Foundation of China for Young Scholars (Grant no. 81600762); the Project of Shanghai Science and Technology (Grant no. 19140900700) and the Shanghai Shenkang Hospital Development Center (Grant no. SHDC12016207).

Competing interests None declared.

\section{Patient consent for publication Obtained.}

Ethics approval This study followed the tenets of the Declaration of Helsinki and was approved by the ethics committee of the Eye and ENT Hospital of Fudan University.

Provenance and peer review Not commissioned; externally peer reviewed.

Data availability statement All data relevant to the study are included in the article or uploaded as supplementary information. Our data are available when all authors agree. Email: doctzhouxintao@163.com.

Open access This is an open access article distributed in accordance with the Creative Commons Attribution Non Commercial (CC BY-NC 4.0) license, which permits others to distribute, remix, adapt, build upon this work non-commercially, and license their derivative works on different terms, provided the original work is properly cited, appropriate credit is given, any changes made indicated, and the use is non-commercial. See: http://creativecommons.org/licenses/by-nc/4.0/.

\section{ORCID iD}

Xingtao Zhou http://orcid.org/0000-0002-3465-1579

\section{REFERENCES}

1 Kawamorita T, Shimizu K, Shoji N. Theoretical study on the need for laser iridotomy in an implantable collamer lens with a hole using computational fluid dynamics. Eye 2017:31:795-801.

2 Kawamorita T, Uozato H, Shimizu K. Fluid dynamics simulation of aqueous humour in a posterior-chamber phakic intraocular lens with a central perforation. Graefes Arch Clin Exp Ophthalmol 2012;250:935-9.

3 Kawamorita T, Shimizu K, Shoji N. Effect of hole size on fluid dynamics of a posteriorchamber phakic intraocular lens with a central perforation by using computational fluid dynamics. Graefes Arch Clin Exp Ophthalmol 2016;254:739-44.

4 Moser CL, Martin-Baranera M, Garat M, et al. Corneal edema and intraocular pressure after cataract surgery: randomized comparison of Healon5 and Amvisc plus. I Cataract Refract Surg 2004;30:2359-65.

5 Ganesh S, Brar S, Pawar A. Matched population comparison of visual outcomes and patient satisfaction between 3 modalities for the correction of low to moderate myopic astigmatism. Clin Ophthalmol 2017;11:1253-63.

6 Cao X, Wu W, Wang Y, et al. Posterior chamber collagen copolymer phakic intraocular lens with a central hole for moderate-to-high myopia: first experience in China. Medicine 2016;95:e4641.

7 Hyun J, Lim DH, Eo DR, et al. A comparison of visual outcome and rotational stability of two types of toric implantable collamer lenses (TICL) : V4 versus V4C. PLoS One 2017; 12:e0183335.

8 Chen X, Miao H, Naidu RK, et al. Comparison of early changes in and factors affecting vault following posterior chamber phakic implantable collamer lens implantation without and with a central hole (ICI V4 and ICI V4C). BMC Ophthalmol 2016;16:161-70 
9 Miao H, Chen X, Tian M, et al. Refractive outcomes and optical quality after implantation of posterior chamber phakic implantable collamer lens with a central hole (ICI V4C). BMC Ophthalmol 2018;18:141-8.

10 Yan Z, Miao H, Zhao F, et al. Two-Year outcomes of Visian implantable collamer lens with a central hole for correcting high myopia. J Ophthalmol 2018;2018:1-9.

11 Alfonso JF, Lisa C, Fernández-Vega Cueto L, et al. Clinical outcomes after implantation of a posterior chamber collagen copolymer phakic intraocular lens with a central hole for myopic correction. J Cataract Refract Surg 2013;39:915-21.

12 Packer M. Meta-Analysis and review: effectiveness, safety, and central Port design of the intraocular collamer lens. Clin Ophthalmol 2016;10:1059-77.

13 Bhandari V, Karandikar S, Reddy JK, et al. Implantable collamer lens V4b and V4c for correction of high myopia. J Curr Ophthalmol 2015;27:76-81.

14 Lee J, Kim Y, Park S, et al. Long-Term clinical results of posterior chamber phakic intraocular lens implantation to correct myopia. Clin Exp Ophthalmol 2016;44:481-7.

15 Igarashi A, Shimizu K, Kamiya K. Eight-Year follow-up of posterior chamber phakic intraocular lens implantation for moderate to high myopia. Am J Ophthalmol 2014; 157:532-9.

16 Lisa C, Naveiras M, Alfonso-Bartolozzi B, et al. Posterior chamber collagen copolymer phakic intraocular lens with a central hole to correct myopia: one-year follow-up. J Cataract Refract Surg 2015;41:1153-9.

17 Kamiya K, Shimizu K, Igarashi A, et al. Posterior chamber phakic intraocular lens implantation: comparative, multicentre study in 351 eyes with low-to-moderate or high myopia. Br J Ophthalmol 2018;102:177-81.

18 Bourne WM, Nelson LR, Hodge DO. Central corneal endothelial cell changes over a ten-year period. Invest Ophthalmol Vis Sci 1997;38:779-82.

19 Shimizu K, Kamiya K, Igarashi A, et al. Long-Term comparison of posterior chamber phakic intraocular lens with and without a central hole (hole ICl and conventional ICI) implantation for moderate to high myopia and myopic astigmatism: consortcompliant article. Medicine 2016;95:e3270.

20 Saxena R, Boekhoorn SS, Mulder PGH, et al. Long-Term follow-up of endothelial cell change after artisan phakic intraocular lens implantation. Ophthalmology 2008;115:608-13.
21 Niu L, Miao H, Han T, Tian H, et al. Visual outcomes of Visian ICl implantation for high myopia in patients with shallow anterior chamber depth. BMC Ophthalmol 2019;19:121-7.

22 Garcia-De la Rosa G, Olivo-Payne A, Serna-Ojeda JC, La G-de, Rosa G, et al. Anterior segment optical coherence tomography angle and vault analysis after toric and nontoric implantable collamer lens V4c implantation in patients with high myopia. $\mathrm{Br} J$ Ophthalmol 2018;102:544-8.

23 Shajari M, Scheffel M, Koss MJ, et al. Dependency of endothelial cell loss on anterior chamber depth within first 4 years after implantation of iris-supported phakic intraocular lenses to treat high myopia. J Cataract Refract Surg 2016;42:1562-9.

24 Kohnen T, Maxwell WA, Holland S. Correction of Moderate to High Myopia with a Foldable, Angle-Supported Phakic Intraocular Lens: Results from a 5-Year Open-Label Trial. Ophthalmology 2016;123:1027-35.

25 Alió JL, Plaza-Puche AB, Cavas F, et al. An angle-supported foldable phakic intraocular lens for correction of myopia: a five-year follow-up. Arch Soc Esp Oftalmol 2017;92:4-11.

26 Jonker SMR, Berendschot TTJM, Ronden AE, et al. Long-Term endothelial cell loss in patients with artisan myopia and artisan toric phakic intraocular lenses: 5- and 10year results. Ophthalmology 2018;125:486-94.

27 Fernández-Vigo Jl, Macarro-Merino A, Fernández-Vigo C, et al. Effects of implantable collamer lens V4c placement on iridocorneal angle measurements by Fourier-domain optical coherence tomography. Am J Ophthalmol 2016;162:43-52.

28 Lee H, Kang SY, Seo KY, et al. Dynamic vaulting changes in V4c versus V4 posterior chamber phakic lenses under differing lighting conditions. Am J Ophthalmol 2014;158:1199-204.

29 Fernandes P, González-Méijome JM, Madrid-Costa D, et al. Implantable collamer posterior chamber intraocular lenses: a review of potential complications. J Refract Surg 2011;27:765-76.

30 Gonvers M, Bornet C, Othenin-Girard P. Implantable contact lens for moderate to high myopia: relationship of vaulting to cataract formation. J Cataract Refract Surg 2003;29:918-24.

31 Zeng Q-Y, Xie X-L, Chen Q. Prevention and management of collagen copolymer phakic intraocular lens exchange: causes and surgical techniques. J Cataract Refract Surg 2015;41:576-84. 